\title{
"Medioglycaemia": A new concept in glycaemic control in intensive care (ICU) units?
}

\author{
Victoria H. Tomlinson ${ }^{1}$, Jane Langley ${ }^{2}$, Andrew G. Meal ${ }^{1}$, Gary G. Adams ${ }^{3 *}$ \\ ${ }^{1}$ Faculty of Medicine and Health Sciences, University of Nottingham, Nottingham, UK; \\ Vicky tomlinson@hotmail.com, Andy.Meal@nottingham.ac.uk \\ ${ }^{2}$ Intensive Care Unit, Lincoln County Hospital, Lincoln, UK; \\ Jane.Langley@homecall.co.uk \\ ${ }^{3}$ Insulin and Diabetes Experimental Research (IDER) Group, Faculty of Medicine and Health Sciences, University of Nottingham, \\ Nottingham, UK; ${ }^{*}$ Corresponding Author: Gary.adams@nottingham.ac.uk
}

Received 20 August 2012; revised 20 September 2012; accepted 30 September 2012

\section{ABSTRACT}

Introduction: Critically ill patients can experience stress-induced hyperglycaemia. Glycaemic control therapy (GCT) is administered to control patients' blood glycaemic levels and reduce the incidence of infection, myocardial infarctions and organ failure. However, there are many factors influencing the effectiveness of glycaemic control for patients. This investigation aimed to review the method of Glycaemic Control Therapy (GCT) used in two hospital settings, to assess the effectiveness of glycaemic control on patients' blood glycaemic levels and examine any barriers that may be in place. Method: A retnrospective audit was carried out on patients' case notes in Intensive Care Units (ICU) within the East Midlands, UK. This method prevents the study outcomes being swayed because GCT has already taken place. To reduce selection bias the most recent available case notes were selected. All the patients who were admitted to these adult ICU's between March and April 2010 had their case notes examined, those who were administered GCT were included in the study, this involved $\mathbf{7 9}$ from Hospital $A$ and $\mathbf{5 0}$ from Hospital B. The patients' notes were retrospectively audited. Results: Different glycaemic control protocols were being implemented in each hospital, despite both belonging to the same ICU network. In most incidences, regardless of age, diabetes status or diagnosis, patients were administered the same sliding scale insulin (SSI). It was also found that GCT commenced for $41.9 \%(n=52)$ of ICU patients (across both Hospitals) when glycaemic levels were below the established threshold of $10 \mathrm{mmol} / \mathrm{L}$. Additionally, a new glycae- mic range has been discovered, where $\mathbf{8 8 . 3 \%}$ ( $n=113$ ) of patients (across both Hospitals) receiving GCT were not controlled in hypoglycaemia, normoglycaemia or hyperglycaemia. They had mean blood glycaemic levels maintained between 5.6 - $9.9 \mathrm{mmol} / \mathrm{L}$, now being described as medioglycaemia. Conclusions: The majority of patients receiving GCT were controlled in medioglycaemia and therefore a new comprehensive guideline needs to be developed incorporating this new range. Recommendations also need to be established to adapt the titration regimen to individual patients, to improve the effectiveness and safety of glycaemic control.

Keywords: Glycaemic Control; Intensive Care (ICU) Units; Medioglycaemia; Tight Glycaemic Control

\section{INTRODUCTION}

Hyperglycaemia increases patients' risk of myocardial infarction, cardiac failure and stroke, emphasising the need for effective Glycaemic Control Therapy (GCT) to be implemented [1].

\subsection{Stress-Induced Hyperglycaemia}

The endocrine cells in the pancreas normally maintain blood glycaemic levels between 3.9 - $5.5 \mathrm{mmol} / \mathrm{litre}$. However, the body's neuro-endocrine functions can be disrupted in critically ill patients causing blood glycaemic levels to increase above normal ranges, known as stress-induced hyperglycaemia [1-3]. Stress-induced hyperglycaemia can occur in critically ill patients who were previously not diabetic, but consequently may be susceptible to the same adverse health complications a person with diabetes mellitus experiences [4]. Controlling blood glycaemia reduces the incidence of severe infections, 
sepsis, myocardial infarctions and multiple organ failure emphasising the need for effective glycaemic control [1,5-7].

\subsection{Landmark in the Development of Tight Glycaemic Control}

The introduction of Tight Glycaemic Control (TGC) by Van den Berghe et al.'s 2001 randomised controlled trial, challenged the then current belief that stress-induced hyperglycaemia was beneficial towards the patients. When patients' blood glycaemic levels were maintained with conventional treatment between $180-200$ $\mathrm{mg} / \mathrm{dL}(10-11.1 \mathrm{mmol} / \mathrm{L})$ the patients mortality rate was $8 \%$ (63 out of 783), compared to the TGC group maintaining glycaemic levels of $4.4-6.1 \mathrm{mmol} / \mathrm{L}$ (80 - 110 $\mathrm{mg} / \mathrm{dL}$ ) where the mortality rate was $4.6 \%$ (35 out of 765 ). Reduced organ failure and infection rates were also found [4]. In response to these findings, hundreds of ICU's attempted to apply TGC to their practice [8].

\subsection{Difficulties Found with the Tight Glycaemic Control Protocol}

It was discovered that many nurses found the TGC protocol complex to implement [9]. An audit found that only $32.8 \%(\mathrm{n}=11,916)$ of patient's blood glycaemic levels were maintained within the TGC target range of $4.4-6.1 \mathrm{mmol} / \mathrm{L}$, compared to $81.9 \%(\mathrm{n}=29,739)$ of blood glycaemic values maintained in the Surviving Sepsis target range of $<150 \mathrm{md} / \mathrm{dL}(8.3 \mathrm{mmol} / \mathrm{L})$, illustrating the problem of adherence to TGC. However, timely blood glycaemic measurements and accurate adjustments could result in less variability and hypoglycaemic incidences $[10,11]$.

\subsection{Further Investigations}

A large randomised trial carried out by NICE-SUGAR investigators argued that TGC increases mortality rates and the number of second-degree hypoglycaemia $(\leq 2.2$ $\mathrm{mmol} / \mathrm{L}$ ) events experienced [12]. Additionally, in 2006 Van den Berghe et al.'s landmark study was repeated. This trial, however, failed to demonstrate the benefit of the intensive insulin therapy on mortality [13]. Furthermore, the SepNet trial studied the use of TGC in patients with severe sepsis, but was stopped due to many hypoglycaemic episodes [14]. Yet, the Surviving Sepsis Campaign still recommend that TGC is administered because the desirable effects of this implementation outweigh the unwelcome effects [15].

\subsection{Current Recommendations}

There are many conflicting recommendations in the literature for ICU practitioners to implement. There is no protocol in place that provides the ICU practitioners with guidelines that address the variety of patient diagnoses that affect glycaemic control $[16,17]$. This has emphasised the need for further investigations in order to determine what is currently implemented and its effectiveness.

\section{METHOD}

\subsection{Aims}

An Audit was used to examine what GCT is currently implemented in ICU settings and its impact on patient's blood glycaemic levels.

Null Hypothesis $\left(\mathrm{H}_{0}\right)$ : There would be no difference in the glycaemic control protocols used between the two hospitals. This was hypothesised because the hospitals belong to the same ICU network.

\subsection{Objectives}

- To review the method of glycaemic control used in both hospital settings.

- To assess the effectiveness of glycaemic control on patients' blood glycaemic levels.

- To examine any barriers that may be in place affecting glycaemic control.

\subsection{Study Design}

Patients' case notes were examined retrospectively, using a base line audit with self constructed standards.

The patients' case notes were examined and grouped into the type of GCT administered. Recorder blood glycaemic levels were collected to determine 1) what level GCT commenced; 2) the efficiency of blood glycaemic control (by calculating the mean blood glycaemic level) to group into a) Hyperglycaemia $(\geq 10 \mathrm{mmol} / \mathrm{L})$ or b) below hyperglycaemia. Other information recorded included the patient demographics, time spent in ICU until GCT was commenced, overall length of stay and medications such as the type of insulin and nutritional methods administered.

\subsection{Sample}

The patients' case notes that were admitted to each ICU over the two months: March and April of 2010, were examined. In "Hospital A" this was 169 case notes and in "Hospital B" this was 157 case notes.

\subsection{Data Collection}

The data were collected over a period of 12 weeks. The inclusion criteria were for all the patients to have been admitted to Adult ICU and to have had GCT whilst 
in ICU between 1st March 2010 and 30th April 2010 (61 days). Patients were excluded if they did not receive any GCT.

\subsection{Ethical Considerations}

Ethical approval is not required for a clinical audit, although, audit registrations were submitted to both hospitals and were approved by the Audit Clerks' covering the Critical Care or diabetes directorate. Patient confidentiality was maintained by making patients fully anonymous and examining their case notes in secure settings [18].

\subsection{Data Analysis}

The analytical statistics have been calculated in PASW Statistics 18. Cross tabulation Chi-square statistics were calculated to determine the significance of proportions of patients mean blood glycaemic levels being maintained in either hyperglycaemia ( $\geq 10 \mathrm{mmol} / \mathrm{L}$ ) or below. Fisher's Exact $p$ value was then used to determine the exact probability of the outcomes in a $2 \times 2$ table because of small sample sizes.

\section{RESULTS}

\subsection{Study Population}

The number of patients who fitted the inclusion criteria were 79 in Hospital A, 48 male:31 female, compared to 50 in Hospital B, 33 male:17 female. Both hospitals had the same age range of $16-87$ years, but the mean age in Hospital A was 58 years compared to 63 years in Hospital B.

The majority of patients receiving GCT in these hospital samples were non-diabetic, this included $75.9 \%$ (n $=60)$ of Hospital A's patients and $60.0 \%(\mathrm{n}=30)$ in Hospital B. Type 1 Diabetic Mellitus patients' made up 5.1\% $(n=4)$ of patients in Hospital A and $12.0 \%(n=6)$ in Hospital B, whilst Type 2 Diabetes Mellitus patients' made up $19.0 \%(n=15)$ of patients in Hospital $A$ and $28.0 \%(\mathrm{n}=14)$ in Hospital B.

\subsection{Glycaemic Control Therapy}

In Hospital A, 93.7\% $(\mathrm{n}=73)$ of patients were put on Actrapid intravenous SSI. A further 3.8\% $(n=3)$ of patients received an Actrapid bolus and the final 2.5\% $(\mathrm{n}=$ 2) of patients were on anti-diabetic tablets. The number of patients on GCT now equals 78 because of missing documentation for one Type 1 diabetic patient (excluded hereafter).

In Hospital B, 92\% $(n=46)$ of patients were put on Actrapid intravenous SSI. A further $6 \%(n=3)$ of patients were on Actrapid intravenous SSI converted to Actrapid subcutaneous SSI and $2 \%(n=1)$ were on Ac- trapid subcutaneous SSI.

\subsection{Blood Glycaemic Control}

Table 1 shows the proportions of patients within each hospital controlled in hyperglycaemic ranges $(\geq 10$ $\mathrm{mmol} / \mathrm{L}$ ) or below this. These two ranges have been chosen because the literature suggests that GCT commences when a patient becomes hyperglycaemic (10 $\mathrm{mmol} / \mathrm{L})[12,16]$.

The Fisher's exact Chi-square test suggests a $77 \%$ probability (2-sided) that the proportional difference may be as a result of chance and therefore not statistically significant. However, the distribution of patients still indicates a trend that the majority of patients have GCT controlling their mean blood glycaemic levels below hyperglycaemia.

Examination of the data indicated that the two hospitals were controlling their patients below hyperglycaemia $(\leq 9.9 \mathrm{mmol})$ but above normoglycaemia $(\geq 5.6 \mathrm{mmol} / \mathrm{L})$. Thus, the majority of patients who required GCT in ICU were being controlled in an uncategorised blood glycaemic range $(5.6$ - $9.9 \mathrm{mmol} / \mathrm{L})$.

This uncategorised blood glycaemic range (5.6 - 9.9 $\mathrm{mmol} / \mathrm{L}$ ) has not been previously published and therefore, will be referred to hereinafter as medioglycaemia [19].

It was found that $88.3 \%(\mathrm{n}=113)$ of patients (across Hospital A and B) receiving GCT had mean blood glycaemic levels maintained in the medioglycaemic range (5.6 - $9.9 \mathrm{mmol} / \mathrm{L}$ ), see Table 2. None of the patients had mean blood glycaemic levels of $\leq 3.3 \mathrm{mmol} / \mathrm{L}$ (hypoglycaemic) [19].

Figure 1 compares the patients mean blood glycaemic level with the glycaemic level when GCT was commenced on ICU in both hospitals. The horizontal line on the graph divides the patients into the groups of patients, who commenced GCT when the patient's blood glycaemic was $10 \mathrm{mmol} / \mathrm{L}$.

In Hospital A, 53.3\% $(n=40)$ began GCT when the patient's glycaemic levels were $\geq 10 \mathrm{mmol} / \mathrm{L}$, whilst $46.7 \%(\mathrm{n}=35)$ were commenced GCT when they were $\leq 9.9 \mathrm{mmol} / \mathrm{L}$. The two normoglycaemic patients and one other patient are not charted because the exact level GCT commenced was unknown.

In Hospital B, 65.3\% $(\mathrm{n}=32)$ were commenced on GCT when their glycaemic levels were $\geq 10 \mathrm{mmol} / \mathrm{L}$. However, $34.7 \%(\mathrm{n}=17)$ were still commenced on GCT when their blood glycaemic level was $\leq 9.9 \mathrm{mmol} / \mathrm{L}$. One patient has also not been charted because the exact time their GCT commenced was not documented.

Figure 2 displays the number of hours the patient was in ICU before GCT commenced. The hours were rounded up to the nearest whole hour. In Hospital A the number of hours ranged from $1-157$ hours compared to 
Table 1. Fisher's exact test statistics examining the patients mean blood glycaemic level across both hospital sites.

\begin{tabular}{|c|c|c|c|}
\hline \multicolumn{4}{|c|}{ Mean Blood Glycaemic Levels } \\
\hline & Below Hyperglycaemia & Hyperglycaemic & \\
\hline & $9.9 \mathrm{mmol} / \mathrm{L}$ and below & $\geq 10.0 \mathrm{mmol} / \mathrm{L}$ & Fisner's Exact Iest \\
\hline & Number (\%) & Number (\%) & $\begin{array}{c}\text { Exact Significant } 2 \text { sided } \\
\text { p value }\end{array}$ \\
\hline Hospital A & $69(88.5)$ & $9(11.5)$ & \\
\hline Hospital B & $46(92.0)$ & $4(8)$ & \\
\hline Total & $115(89.8)$ & $13(10.2)$ & 0.765 \\
\hline
\end{tabular}

Table 2. Patients mean blood glycaemic level divided into the blood glycaemic ranges.

\begin{tabular}{cccc}
\hline & & Mean Blood Glycaemic Levels & \\
& Normoglycaemia & Medioglycaemia $_{\text {New Range }}^{*}$ & Hyperglycaemia \\
& $\mathbf{3 . 9}-\mathbf{5 . 5} \mathbf{~ m m o l} / \mathbf{L}$ & $\mathbf{5 . 6}-\mathbf{9 . 9} \mathbf{~ m m o l} / \mathbf{L}$ & $\mathbf{1 0} \mathbf{m m o l} / \mathbf{L}$ \\
Number (\%) & Number (\%) & Number (\%) & $9(11.5)$ \\
Hospital A & $2(2.6)$ & $67(85.9)$ & $4(8.0)$ \\
Hospital B & $0(0)$ & $46(92.0)$ & $13(10.1)$ \\
\hline Total & $2(1.6)$ & $113(88.3)$ &
\end{tabular}

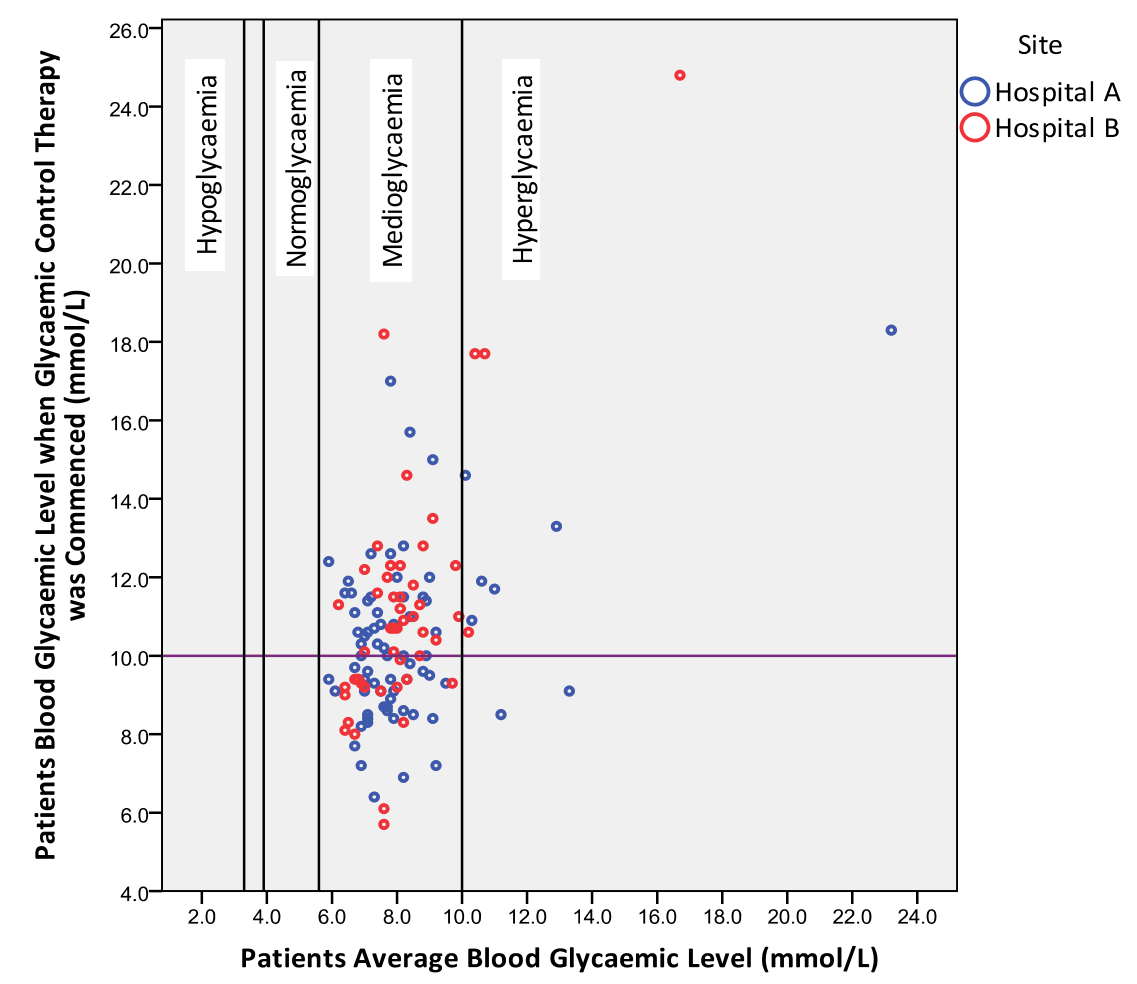

Figure 1. Compares the patients mean blood glycaemic level with the glycaemic level when Glycaemic Control Therapy was commenced on Intensive Care Unit in both hospitals. The horizontal line on the graph divides the patients into the groups of patients, who commenced Glycaemic Control Therapy when the patient's blood glycaemic was 10 $\mathrm{mmol} / \mathrm{L}$. 


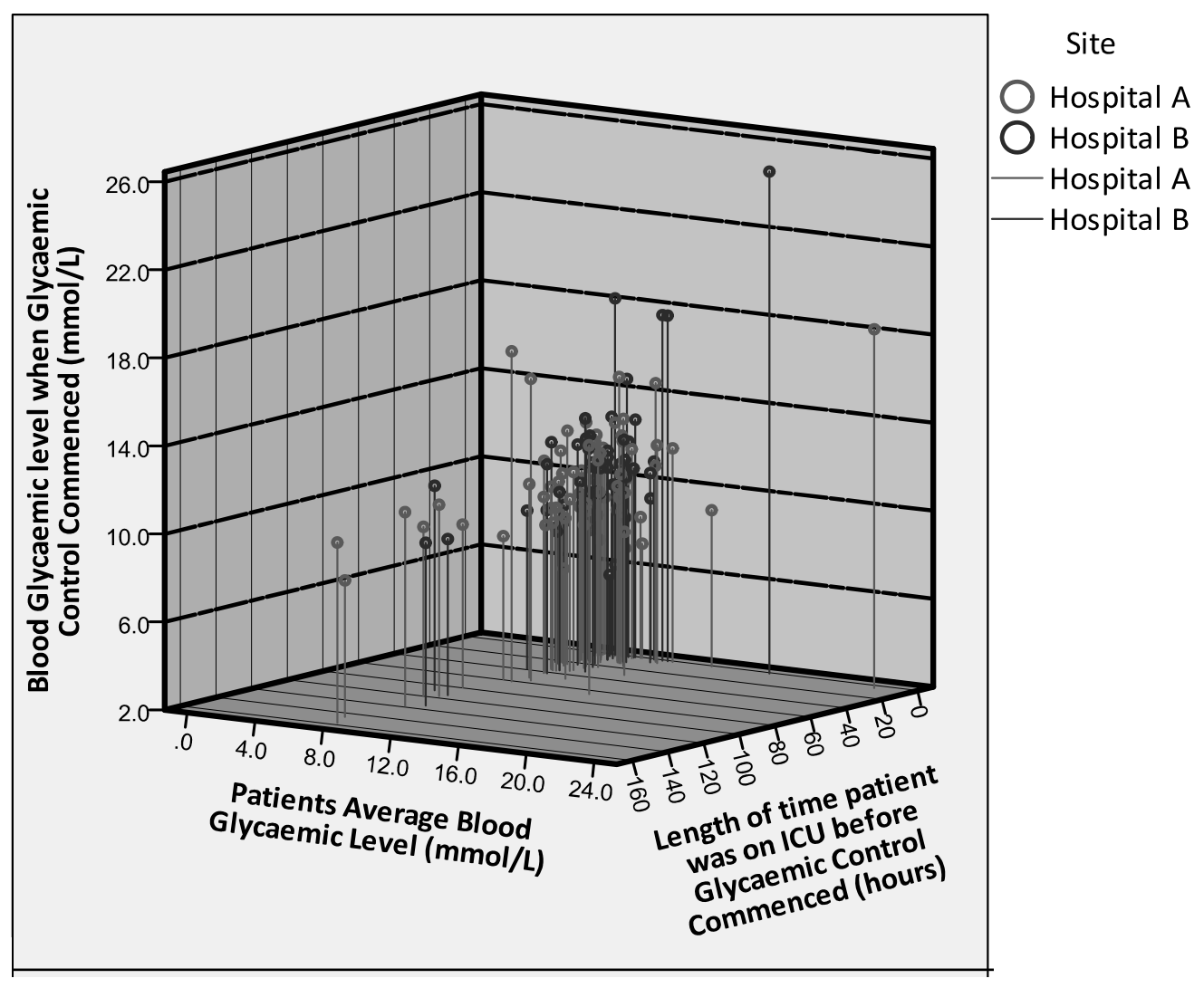

Figure 2. Examining the patient's mean blood glycaemic level with their actual blood glycaemic level when Glycaemic Control Therapy was commenced and the number of hours the patient was on Intensive Care Unit before Glycaemic Control Therapy was initiated.

1 - 114 in Hospital B. The majority of patients were commenced on GCT between 1 - 10 hours, including $46.7 \%(\mathrm{n}=35)$ of patients in Hospital A compared to $55.1 \%(\mathrm{n}=27)$ in Hospital B (Table 3).

\section{DISCUSSIONS}

Prior to the discussion of the results, a brief synopsis is provided of the protocols used in each of the respective hospitals because of the different glycaemic control protocols implemented, despite both belonging to the same ICU network. In most incidences, regardless of age, diabetes status or diagnosis, patients were administered the same SSI.

\subsection{Hospital Protocols}

Hospitals A and B have different Actrapid intravenous sliding scales with separate titrations, whilst Hospital B also administered a subcutaneous SSI infrequently, but on an irregular basis.

Despite both hospitals belonging to the same ICU network, Hospital A had documented glycaemic control ranges of 1) 4 - $8.3 \mathrm{mmol} / \mathrm{L}$; 2) $4-6.9 \mathrm{mmol} / \mathrm{L}$ and 3$) 8$ $11 \mathrm{mmol} / \mathrm{L}$ compared to $4-6.1 \mathrm{mmol} / \mathrm{L}$ in Hospital B.
Additionally, Hospital B's titration regimen was developed in 2001, whilst Hospital A's protocol and regimens were last updated in 2008.

Thus, a number of issues have arisen, as a result of investigations in to these ITUs. 1) A variety of insulin protocols are available, which may or may not be implemented; 2) Insulin infusions are irregularly implemented, between and within these hospitals; 3) Protocols/regimens are out of date, which could impact the care provided.

\subsection{Discussion}

It was hypothesised that there would be no difference in the glycaemic control protocols used between the two hospitals but this audit has raised many questions surrounding the administration of GCT.

Figure 1 illustrates the hyperglycaemic threshold of $\geq 10 \mathrm{mmol} / \mathrm{L}$ [11] recommended by the NICE-SUGAR Investigators, [12] with a horizontal line. It shows that $41.9 \%(\mathrm{n}=52)$ across Hospital A \& B were commenced on GCT below this threshold of $\leq 10 \mathrm{mmol} / \mathrm{L}$, despite the recommendations in the literature. On the other hand, the ADA recommends a threshold of $7.8 \mathrm{mmol} / \mathrm{L}$ for nondiabetic patients because they suggest this level is hyper- 
Table 3. The majority of patients in both hospitals were commenced on Glycaemic Control Therapy between $1-10$ hours with $46.7 \%(n=35)$ in Hospital A compared to $55.1 \%(n=27)$ in Hospital B.

\begin{tabular}{cccccccccccccccc}
\hline & $1-10$ & $11-20$ & $21-30$ & $31-40$ & $41-50$ & $51-60$ & $61-70$ & $71-80$ & $81-90$ & $91-100$ & $111-120$ & $141-150$ & $151-160$ & Total \\
\hline Hospital A & 35 & 9 & 9 & 8 & 3 & 3 & 2 & 1 & 0 & 2 & 1 & 1 & 1 \\
Hospital B & 27 & 7 & 7 & 4 & 1 & 0 & 0 & 0 & 1 & 1 & 1 & 0 & 0 & 49 \\
Total & 62 & 16 & 16 & 12 & 4 & 3 & 2 & 1 & 1 & 3 & 2 & 1 & 1 & 124 \\
\hline
\end{tabular}

glycaemic for a non-diabetic [20]. While $8.3 \mathrm{mmol} / \mathrm{L}$ $(150 \mathrm{mg} / \mathrm{dL})$ is suggested to reduce the probability of sepsis, [15] yet there is little supporting evidence for this. As a result of this contradictory information, many healthcare practitioners are unsure as to which form of treatment is beneficial to the care and QoL for the patient.

This contradictory information could have resulted in many non-diabetics being commenced on insulin, when their glycaemic levels were within the medioglycaemic range. Both hospitals verbally stated, but had not previously documented, that they commenced their insulin therapy at blood glycaemic values of $\geq 10 \mathrm{mmol} / \mathrm{L}$ (hyperglycaemia), although, $41.9 \%(n=52)$ were commenced at values $\leq 9.9 \mathrm{mmol} / \mathrm{L}$. The dichotomy between what is stated and actually delivered could indicate that hyperglycaemia levels may in fact be $<10 \mathrm{mmol} / \mathrm{L}$, especially as $71.4 \%(n=90)$ of the patients in Hospital A and $60.0 \%(\mathrm{n}=30)$ of the patients in Hospital B were non-diabetics requiring GCT. This may indicate that healthcare professionals are inappropriately and ineffectively diagnosing hyperglycaemia.

Thus, the blood glycaemic level where GCT is commenced needs to be re-evaluated.

It has been found that patients who were commenced earlier on GCT were those who had had hyperglycaemic blood glycaemic values early on in their admission (Figure 2). The patients who were commenced on GCT later than these patients seemed to have blood glycaemic levels that were medioglycaemic. It seems that in both hospitals, a decision was made to control their blood glycaemic levels because they remained constantly above normal.

The glycaemic control results have shown that there are now four glycaemic groups: 1) hypoglycaemia; 2) normoglycaemia; 3) medioglycaemia and 4) hyperglycaemia, with the introduction of this nascent medioglycaemic range $(5.6-9.9 \mathrm{mmol} / \mathrm{L})$. What is important to establish, however, is the distinction between medioglycaemia triggering GCT, and actually having blood glucose maintained in the medioglycaemic range.

We shall now explore each of these points in turn:

1) If medioglycaemia is the trigger for treatment, this means that clinicians perceive a need for treatment, but there is no empirical evidence for doing so;
2) If blood glucose is being maintained within the medioglycaemic range, this reflects the wide variety of recommended maintenance values identified within the literature. However, there is a lack of consistency as to the ideal maintenance range.

The American Association of Clinical Endocrinologists (AACE) \& American Diabetes Association (ADA) still fail to provide health professionals with a protocol that addresses the variety of patients admitted to ICU. An established glycaemic control protocol is, nevertheless, still required to accommodate the needs of patients admitted with different diagnoses [17].

Health professionals fear the implications of hyperglycaemia and more so hypoglycaemia [13]. However, if patients are maintained within this medioglycaemic range with the development of a new TGC titration regimen and protocol, healthcare professionals could reduce the glycaemic variance experienced by the patients and minimise still further the risks of hyperglycaemic and hypoglycaemic events [21].

Defining normoglycaemia is also difficult. The normoglycaemic level used for this audit follows the ADA recommendations of between $3.9-5.5 \mathrm{mmol} / \mathrm{L}$ [22,23] although other published blood glycaemic ranges that are described as normoglycaemic include $4.4-6.1 \mathrm{mmol} / \mathrm{L}$ [13], $4.4-5.6 \mathrm{mmol} / \mathrm{L}$ [2] and $3.8-7.2 \mathrm{mmol} / \mathrm{L}$ [24]. This therefore, indicates inconsistencies surrounding the definition of normoglycaemia and this will affect and determine titration regimens.

This audit confirms clinicians are maintaining glycaemia within recommended ranges that together constitute medioglycaemia. However the ranges are wide and this is reflected in our data. Between one-third and onehalf of all ICU patients on GCT have their treatment instigated below the established threshold of $10 \mathrm{mmol} / \mathrm{L}$. Does this mean this level should be re-evaluated/ amended or are clinicians inadvertently responding to other cues about patients' conditions, which are not recorded in the audit data?

Neither our data nor the literature can answer this question. Therefore, a qualitative study which addresses this question needs to be undertaken to try to establish why clinicians instigate GCT treatment below $10 \mathrm{mmol} /$ L. 


\subsection{Limitations}

Only selecting 2 months of data has meant that only a snap-shot of ICU glycaemic control data has been collected and therefore cannot be generalised across the whole year, because the ICU environment is constantly changing with a variety of patients, diagnoses and rotating health professionals.

Some patients' notes were poorly documented, thus hindering the evaluation of what the patients may have been administered and how they were controlled.

Mean blood glucose levels were calculated for each patient however, mean values can be distorted by extreme values. Other metrics could have reflected different blood glycaemic values [25].

\section{CONCLUSIONS}

The retrospective audit carried out on patients' case notes across two ICU's in the East Midlands, UK has shown that different glycaemic control protocols were implemented in each intensive care setting, despite both belonging to the same ICU network. In most instances, regardless of age, diabetes status or diagnosis, patients were administered the same SSI, medications were not taken into account for protocol titrations and Actrapid was the only insulin used for SSI.

The authors also established that the majority of patients receiving GCT were not controlled in hypoglycaemia, normoglycaemia or hyperglycaemia, but within the nascent medioglycaemic range of $5.6-9.9 \mathrm{mmol} / \mathrm{L}$.

\section{ACKNOWLEDGEMENTS}

Dr. Alan Liddle, Consultant Intensivist, Lincolnshire County Hospital PCT.

Dr. David Sperry, Consultant Intensivist, Nottingham University Hospitals NHS Trust.

\section{REFERENCES}

[1] Marik, P.E. and Preiser, J.C. (2010) Towards understanding tight glycemic control in the ICU: A systematic review and metaanalysis. Chest, 137, 544-551. doi:10.1378/chest.09-1737

[2] Spuhler, V.J. and Veale, K.V. (2007) Tighten up glycaemic control: Aggressive insulin therapy in critical care settings is the latest method for reducing mortality rates. Critical Care Insider, 37, 10-13.

[3] Sage, D. (2009) Glycaemic control: It's not just about diabetes. Academy of Medical-Surgical Nurses, 18, 11-15.

[4] Van den Berghe, G., Wouters, P., Weekers, F., et al. (2001) Intensive Insulin Therapy in Critically Ill patients. The New England Journal of Medicine, 345, 1359-1366. doi:10.1056/NEJMoa011300

[5] Parsons, P. and Watkinson, P. (2007) Blood glucose con- trol in critical care patients-A review of the literature. British Association of Critical Care Nurses, 12, 202-210.

[6] Pielmeier, U., Andreassen, S., Juliussen, B., Chase, J.G., Nielsen, B.S. and Haure, P. (2010) The Glucosafe system for tight glycaemic control in critical care: A pilot evaluation study. Journal of Critical Care, 25, 97-104. doi:10.1016/j.jcrc.2009.10.003

[7] Gerstein, H.C. (2010) Prevention of the consequences of diabetes-A commentary. In: Herman, W.H., Kinmonth, A.L., Wareham, N.J. and Williams, R., Eds., The Evidence Base for Diabetes Care, 2nd Edition, Wiley-Blackwell, Oxford, 266-272.

[8] Bellomo, R. (2009) What is a nice-sugar for patients in the intensive care unit? Mayo Clinic Procedures, 84, 400402.

[9] Harper, J. (2007) BAPEN Symposium 6 on "Inflammation technology: Putting theory into practice" Glucose control in the intensive care unit: How it is done. Proceedings of the Nutrition Society, 66, 362-366. doi:10.1017/S0029665107005629

[10] Siegelaar, S.E., Holleman, F., Hoekstra, J.B.L. and Hans DeVries, J. (2010) Glucose variability; Does it matter? Endocrine Reviews, 31, 171-182. doi:10.1210/er.2009-0021

[11] Anabtawi, A., Hurst, M., Titi, M., Patel, S., Palacio, C. and Rajamani, K. (2010) Incidence of hypoglycemia with tight glycemic control protocols: A comparative study. Diabetes Technology \& Therapeutics, 12, 635-639. doi:10.1089/dia.2010.0009

[12] Investigators, N.-S. (2009) Intensive versus conventional glucose control in critically ill patients. The New England Journal of Medicine, 360, 1283-1297. doi:10.1056/NEJMoa0810625

[13] Van den Berghe, G., Wilmer, A., Hermans, G., et al. (2006) Intensive insulin therapy in the medical ICU. New England Journal of Medicine, 354, 449-461. doi:10.1056/NEJMoa052521

[14] Webater, N.R. and Galley, H.F. (2009) Does strict glucose control improve outcome? British Journal of Anaesthesia, 103, 331-334. doi:10.1093/bja/aep226

[15] Dellinger, R.P., Levy, M.M., Carlet, J.M., et al. (2008) Surviving sepsis campaign: International guidelines for management of severe sepsis and septic shock. Critical Care Medicine, 36, 296-327. doi:10.1097/01.CCM.0000298158.12101.41

[16] Moghissi, E.S., Korytkowski, M.T., DiNardo, M., et al. (2009) American association of clinical Endocrinologists (AACE) and the American diabetes association (ADA) consensus statement on inpatient glycemic control. AACE/ ADA Consensus Statement. Endocrine Practice, 15, 1-11. doi:10.4158/EP09102.RA

[17] Corbin, A.E., Carmical, D., Goetz, J.A., et al. (2010) One institution's experience in implementing protocols for glycemic management. Dimensions of Critical Care Nursing, 29, 167-170. doi:10.1097/DCC.0b013e3181de98da

[18] Ghosh, R. (2009) Clinical audit for doctors. Developmedica, Nottingham.

[19] Tomlinson, V. (2011) Unpublished Thesis. 
[20] American Diabetes Association (2010) Standards of medical care in diabetes. Diabetes Care, 33, S11-S61. doi:10.2337/dc10-S011

[21] Meyfroidt, G., Keenan, D.M., Wang, X., Wouters, P.J., Veldhuis, J.D. and Van den Berghe, G. (2010) Dynamic characteristics of blood glucose time series during the course of critical illness: Effects of intensive insulin therapy and relative association with mortality. Critical Care Medicine, 38, 1021-1029. doi:10.1097/CCM.0b013e3181cf710e

[22] American Diabetes Association (2011) Diagnosis and classification of diabetes mellitus. Diabetes Care, 34, S62-S69.

[23] American Diabetes Association (2011) Standards of medical care in diabetes. Diabetes Care, 34, S11-S61. doi:10.2337/dc11-S011

[24] Shogbon, A.O. and Levy, S.B. (2010) Intensive glucose control in the management of diabetes mellitus and inpatient hyperglycemia. American Journal of Health-System Pharmacy, 67, 798-805. doi:10.2146/ajhp090211

[25] Mackenzie, I.M.J., Whitehouse, T. and Nightingale, P.G. (2011) The metrics of glycaemic control in critical care. Intensive Care Medicine, 37, 435-443. doi:10.1007/s00134-010-2103-2

\section{LIST OF ABBREVIATIONS}

BG:

DKA:

DM:

Hyperglycaemia:

Hypoglycaemia:

ICU:

Medioglycaemia:

$\mathrm{mmol} / \mathrm{L}$

TGC:

T1DM:

T2DM:
Blood Glucose

Diabetic Ketoacidosis

Diabetes Mellitus

Glycaemic levels are $\geq 10 \mathrm{mmol} / \mathrm{L}$

Glycaemic levels are $\leq 3.3 \mathrm{mmol} / \mathrm{L}$ Intensive Care Unit

Glycaemic levels between 5.6 - 9.9

Tight Glycaemic Control

Type 1 Diabetes Mellitus

Type 2 Diabetes Mellitus

\section{IMPLICATIONS FOR CLINICAL PRACTICE}

The research has shown that different glycaemic control protocols were implemented in each intensive care setting, despite both belonging to the same ICU network.

Patients were administered the same SSI, regardless of age, diabetes status or diagnosis. Patient medications

were not taken into account for protocol titrations.

Actrapid was the only insulin used for SSI despite the clear need for individualised care. The majority of patients receiving GCT were not controlled in hypoglycaemia, normoglycaemia or hyperglycaemia, but within the posited medioglycaemic range of $5.6-9.9 \mathrm{mmol} / \mathrm{L}$.

\section{FUTURE WORK}

More information needs to be gathered from other hospitals, so that the development of different titration regimens for specific diagnostic groups can advance. The glycaemic level that GCT should be commenced needs to be re-evaluated as there are inconsistencies surrounding the definition of normoglycaemia.

The authors are now investigating glycaemic therapies and the ranges, therein. In addition, they are also investigating different insulin administrations because the audit found that the only insulin to be used for SSI within each ICU was Actrapid. 